Anaesthesist 2017 - 66:477-478

DOI 10.1007/s00101-017-0340-2

Online publiziert: 28. Juni 2017

(c) Springer Medizin Verlag GmbH 2017

CrossMark

\section{P. Mirtschink ${ }^{1}$ - S. N. Stehr}

${ }^{1}$ Institut für Klinische Chemie und Laboratoriumsmedizin, Universitätsklinikum Carl Gustav Carus, Dresden, Deutschland

${ }^{2}$ Klinik und Poliklinik für Anästhesiologie und Intensivtherapie, Universitätsklinikum Leipzig AöR, Leipzig, Deutschland

\title{
Klassische Betrachtung der septischen Kardiomyopathie schärfen
}

Das Auftreten einer infektionsassoziierten Organdysfunktion ist nach der neuen Definition in das Zentrum der Diagnosestellung der Sepsis gerückt [1]. In dieser Ausgabe von Der Anaesthesist beschreiben Martin et al. die Pathophysiologie, Diagnose und Therapie der septischen Kardiomyopathie als eine spezielle Form des infektionsassoziierten Organversagens mit dem klassischen Leitsymptom einer reversiblen Myokarddysfunktion [2].

Die erste wissenschaftlich systematische Untersuchung der septischen Kardiomyopathie wird der Arbeitsgruppe um Margaret Parker und Joseph Parrillo zugeschrieben [3]. Im Jahr 1982 fassten sie Messwerte von 20 Patienten mit septischem Schock zusammen. Bei allen Patienten wurden zur Abschätzung der kardialen Funktion wiederholt aufwendige nuklearmedizinische Messungen der Ejektionsfraktion durchgeführt. Bei 13 Patienten schien das Muster der vorübergehenden Reduktion der initial normalen Myokardfunktion mit einer Erholung nach 7 bis 10 Tagen für das Überleben bestimmend zu sein.

Heutzutage ist die Möglichkeit einer wenig invasiven bettseitigen myokardialen Funktionsdiagnostik fast flächendeckend mittels der Echokardiographie vorhanden. Die septische Kardiomyopathie analog zur Herzinsuffizienz allein anhand der Ejektionsfraktion zu identifizieren, ist vermutlich aufgrund der in einer Sepsis begleitenden kardiovaskulären Veränderungen, wie reduzierte Vorlast durch absolute oder relative Hypovolämie, Bedarfstachykardie sowie reduzierte Nachlast durch Reduktion des vaskulären Tonus, nicht möglich.

Diese kardiovaskulären Veränderungen im Rahmen einer Sepsis können sogar, wie Martin et al. zu Recht darauf hinweisen, eine vorhandene septische Kardiomyopathie maskieren. Ein hochgradiger Widerstandsverlust im Rahmen eines septischen Schocks kann dazu führen, dass trotz einer schon reduzierten kontraktilen Funktion der Myozyten die globale Ejektionsfraktion dennoch in der Echokardiographie als normal erscheinen kann. Dieses Phänomen könnte die vielfältigen Diskrepanzen klinischer Studien bei Patienten mit einer Sepsis mit alleiniger Betrachtung der per Echokardiographie gemessenen Herzfunktion erklären. Es gibt Studien mit Hinweisen sowohl für eine positive wie negative prognostische Bedeutung einer septischen Kardiomyopathie. So zeigten Vieillard-Baron et al. in einer kleinen klinischen Untersuchung ein verbessertes Überleben bei Patienten mit reduzierter Ejektionsfraktion [4]. Landesberg et al. fanden hingegen einen klaren negativen prognostischen Zusammenhang zwischen dem Ausmaß einer systolischen und diastolischen echokardiographischen Funktionsreduktion [5]; dagegen zeigte eine Metaanalyse aus 62 Originalarbeiten, dass weder die rechtsnoch linksventrikuläre Ejektionsfraktion einen Einfluss auf das Überleben der Patienten im septischen Schock hatte [6].

Vielleicht muss daher die klassische Betrachtung der septischen Kardiomyopathie geschärft werden. Besser wäre die gemeinsame Evaluation der Kontraktilität sowie der Vor- und Nachlast. Die Autoren schlagen die Bestimmung der von Werdan et al. eingeführten "afterload-related cardiac performance“ (ACP) vor, um zumindest die nachlastbezogene kardiale Leistungsfähigkeit besser darstellen zu können [7]. Eine weitere sinnvolle Methode scheint laut Martin et al. die "Speckle-tracking“Echokardiographie zu sein, da die myokardiale Kontraktion direkt quantifiziert wird und dadurch weniger von Änderungen der Vor- und Nachlast sowie der myokardialen Compliance beeinflusst werden kann.

Besonders spannend sind neuere Studien, die sich mit den molekularbiologischen Effekten der systemischen Inflammation auf das Myokard beschäftigen. Matkovich et al. zeigten an Myokardbiopsien von Patienten mit septischer Kardiomyopathie im Vergleich zu gesunden Probanden, dass am Ende der durch Inflammation angeregten intrazellulären Signalkaskaden eine verminderte Expression von Genen, codierend für mitochondriale und sarkomerische Proteine, steht [8]. Dies ist im Einklang mit den bekannten Veränderungen des Zellmetabolismus (Aktivierung der Glykolyse, Reduktion der Oxidation) und der myokardialen Kontraktilität unter Inflammation. Vielleicht lassen sich hieraus perspektivisch therapeutische, aber auch diagnostische Verfahren ableiten.

Es bleibt die Erkenntnis, dass es zunächst an einer Definition der septischen Kardiomyopathie mangelt. Auf dem Gebiet der klinischen Medizin sind weitere 
Studien zu Diagnostik und Therapie der septischen Kardiomyopathie notwendig. Dasselbe gilt für den Bereich der Grundlagenforschung, wo Pathomechanismen des Zellmetabolismus sowie der Herzmuskelzellkontraktilität im Rahmen der septischen Kardiomyopathie besser verstanden werden müssen, um den Patienten in Zukunft auch präventive und nicht nur symptombasierte Therapien anbieten zu können.

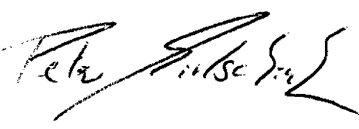

Peter Mirtschink

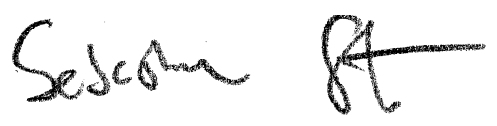

Sebastian Stehr

\section{Korrespondenzadresse}

\section{Prof. Dr. S. N. Stehr, DESA}

Klinik und Poliklinik für Anästhesiologie und Intensivtherapie, Universitätsklinikum Leipzig AöR

Liebigstr. 20, 04103 Leipzig, Deutschland sebastian.stehr@medizin.uni-leipzig.de

Interessenkonflikt. P. Mirtschink und S. N. Stehr geben an, dass kein Interessenkonflikt besteht.

\section{Literatur}

1. Singer M, Deutschman CS, Seymour CW, ShankarHari M, Annane D, Bauer M, Bellomo R, Bernard GR, Chiche JD, Coopersmith CM, Hotchkiss RS, Levy MM, Marshall JC, Martin GS, Opal SM, Rubenfeld GD, van der Poll T, Vincent JL, Angus DC (2016) The third international consensus definitions for sepsis and septic shock (sepsis-3). JAMA 315(8):801-810

2. Martin L, Derwall M, Thiemermann C, Schürholz T (2017) Das Herz in der Sepsis. Molekulare Mechanismen, Diagnose und Therapie der septischen Kardiomyopathie. Anaesthesist. doi:10. 1007/s00101-017-0329-x

3. Parker M, Shelhamer J, Bacharach SL, Green MV, Natanson C, Frederick TM et al (1984) Profound but reversible myocardial depression in patients with septic shock. Ann Intern Med 100(4):483-490

4. Vieillard-Baron A, Schmitt JM, Beauchet A, Augarde R, Prin S Page B, Jardin F (2001) Early Preload Adaptation in Septic Shock?: A Transesophageal Echocardiographic Study. Anesthesiology 94(3): 400-406

5. Landesberg G, Gilon D, Meroz Y, Georgieva M, Levin PD, Goodman S, Avidan A, Beeri R, Weissman C, Jaffe AS, Sprung CL (2012) Diastolic dysfunction and mortality in severe sepsis and septic shock. Eur Heart J33(7):895-903

6. Huang SJ, Nalos M, McLean AS (2013) Is early ventricular dysfunction or dilatation associated with lower mortality rate in adult severe sepsis and septic shock? A meta-analysis. Crit Care 17:R96

7. Werdan K, Oelke A, Hettwer S et al (2011) Septic cardiomyopathy: hemodynamic quantification, occurrence, and prognostic implications. Clin Res Cardiol 100:661-668

8. Matkovich SJ, Al Khiami B, Efimov IR, Evans S (2017) Widespread down-regulation of cardiac mitochondrial and sarcomeric genes in patients with sepsis. Crit Care Med 45:407-414
Hier steht eine Anzeige. Springer 